\title{
Demographic and temporal trends in transgender identities and gender confirming surgery
}

\author{
Ian T. Nolan ${ }^{1}$, Christopher J. Kuhner ${ }^{1}$, Geolani W. Dy ${ }^{2}$ \\ ${ }^{1}$ School of Medicine, ${ }^{2}$ Department of Urology, New York University, New York, NY, USA \\ Contributions: (I) Conception and design: IT Nolan, GW Dy; (II) Administrative support: GW Dy; (III) Provision of study material or patients: All \\ authors; (IV) Collection and assembly of data: All authors; (V) Data analysis and interpretation: All authors; (VI) Manuscript writing: All authors; \\ (VII) Final approval of manuscript: All authors. \\ Correspondence to: Geolani W. Dy, MD. Department of Urology, New York University, 222 East 41st St., 11th Floor, New York, NY 10016, USA. \\ Email: Geolani.Dy@nyumc.org.
}

\begin{abstract}
Transgender and gender non-binary (TGNB) individuals are a growing demographic with unique healthcare needs. Amid changes in public and private insurance coverage of gender confirming surgeries (GCS), utilization of these procedures is increasing. Meanwhile, systemic barriers continue to limit access to gender confirming care and perpetuate health disparities among TGNB individuals. Studies on the prevalence of TGNB identities and utilization of GCS are limited by a lack of gender identity data in population-based surveys and electronic medical records. However, data collection on gender identity is improving, and will be essential for characterizing the healthcare practices and needs of TGNB individuals.
\end{abstract}

Keywords: Transgender; gender confirming surgery; demographic; prevalence

Submitted Nov 08, 2018. Accepted for publication Apr 23, 2019.

doi: 10.21037/tau.2019.04.09

View this article at: http://dx.doi.org/10.21037/tau.2019.04.09

\section{Introduction}

Transgender and gender non-binary (TGNB) people, individuals whose gender identity or expression differs from their sex assigned at birth, are a diverse population with unique healthcare needs. As visibility of TGNB identities increases, so will the demand for high quality transitionrelated and general healthcare for TGNB people. Gender Affirming Surgery, also described as gender confirming surgery (GCS), is one domain of TGNB care that requires specialized urologic expertise. In this review article, we discuss trends in demographics and healthcare utilization among the TGNB population.

\section{Demographics of transgender identities}

Accurate estimates of the United States TGNB population have been challenging to acquire for multiple reasons, including the broad range of terms used to describe transgender identities, and most notably, lack of data collection in population-based surveys. Federallyadministered questionnaires which track the demographics and health of US residents have not historically collected data on gender identity, though some state-level questionnaires have begun to do so $(1,2)$. Utilizing data from the CDC's Behavioral Risk Factor Surveillance System in 19 states, Flores et al. estimated that $0.56 \%$ of US adults $(560$ per 100,000$)$ identify as transgender. In a systematic review of population-based surveys from 2006-2016 that included gender identity questions, Meerwijk et al. estimated that $0.39 \%$ of respondents (390 per 100,000) identified as transgender when this was a categorical option for gender identity. Several smaller studies utilizing limited sources have estimated the U.S, TGNB population as ranging from 38 to 1,647 per 100,000 (3-5). Table 1 summarizes prevalence estimates on transgender identities and gender confirming surgery.

A European systematic review and meta-analysis of "transsexualism" prevalence studies by Arcelus et al. found an overall lower rate of 4.6 per 100,000. However, the 
Table 1 Epidemiology of gender confirming surgery. A summary of the literature's description of the prevalence of TGNB identity, as well as GCS overall and specific procedures in specific populations, is given

\begin{tabular}{lc}
\hline Parameter & $\begin{array}{c}\text { Range of estimated prevalence among } \\
\text { transgender people in the USA, \% (Ref.) }\end{array}$ \\
\hline TGNB identity* & $0.39-2.7^{*}(1,3-6)$ \\
GCS overall & $25-35(7,8)$ \\
GCS in trans men & $42-54(7,8)$ \\
GCS is trans women & $28(7)$ \\
GCS in non-binary & $9(7)$ \\
Genital surgery & $4-13(8,9)$ \\
Trans men & $25-50(1-3)$ \\
Hysterectomy & $14(7)$ \\
Phalloplasty & $3(7)$ \\
Metoidioplasty & $2(7)$ \\
Trans women & $5-13(1-3)$ \\
Non-binary assigned & $1(7)$ \\
male at birth & $8-25(8,9)$ \\
Non-binary assigned & \\
female at birth & $<1(7)$ \\
Hysterectomy in & \\
non-binary & \\
\hline
\end{tabular}

*, estimated percent of total US population. TGNB, transgender and gender non-binary; GCS, gender confirming surgery.

review examined literature published from 1968 to 2014, before much of the recent surge in visibility and acceptance around transgender identities. Additionally, most studies based their estimates upon patients referred to and treated at gender clinics (10).

Regardless of specific prevalence rates, most studies demonstrate two clear trends: (I) growth in the proportion of TGNB self-identifying individuals over time; and (II) a higher proportion of TGNB identities among the younger generations. Flores and colleagues note that their 2016 estimate of the percentage of transgender-identifying adults in the U.S. is double their 2011 estimate, which they attribute to improvement in survey methods (11). Arcelus et al. also describe a general increase in TGNB individuals in Europe within their study period. Greater visibility and acceptance of TGNB individuals, and as a result, greater willingness to self-identify as TGNB and seek transition, may contribute to these trends.

TGNB identification appears to be more common among younger age groups. By the Williams Institute's estimates, TGNB prevalence among adults is highest in the 18-24 years old age group at 700 per 100,000 (0.7\%), compared with 600 per $100,000(0.6 \%)$ in those aged $25-64$ and 500 per $100,000(0.5 \%)$ in those aged 65 and older. Similarly, $42 \%$ of respondents to the 2015 U.S. Transgender Survey (USTS) were between the ages of 18-24, although the surveyed sample is likely skewed based on online survey distribution. The USTS was distributed by the National Center for Transgender Equality and is the largest and most impactful survey to date assessing the needs of the TGNB community, including 27,715 respondents from across the U.S. A recent study of high-school students in Minnesota reported exceptionally high rates of TGNB identities, as high as 2,700 per 100,000 (6). This voluntary survey was administered to 9th and 11th graders in 2016 by the Minnesota Department of Education. Although the Minnesota study is an outlier in its high prevalence rates, it is among the most recent and targets a younger demographic than other prevalence studies, and thus may be indicative of future trends in TGNB identity prevalence.

Evidence supports that TGNB people begin to identify as TGNB when they are at a relatively young age. According to USTS results, by age 20,94\% of respondents began to feel that their gender was different from the sex assigned at birth, $73 \%$ of respondents began to think they were transgender, and $52 \%$ began to tell others that they were transgender (12). These findings were consistent across respondent age groups, which may suggest that reports of growing TGNB prevalence could be attributed to increased awareness, acceptance and self-reporting of TGNB identities among younger generations than an actual increase in prevalence.

Regarding relative prevalence of specific identities, two main trends are described widely in the literature. First, transgender females (females assigned 'male' at birth) are usually identified at higher rates than transgender males (males assigned 'female' at birth). In some studies, the proportion of transgender women to men is as high as 2:1 $(6,10)$. However, these ratios should not be taken as a definitive indication of actual population sizes, given the limitations in methodology used to record them. Many studies survey subjects enrolled in plastic surgery, endocrinology, or primary care clinics, and as such only reflect populations that are well-integrated into existing healthcare systems and accessing care. Furthermore, some 
estimates derived from global data, and definitions of transgender and non-binary identities vary substantially across cultures (10).

Another notable demographic trend is the high prevalence of non-binary identities. "Non-binary" is generally used as an umbrella term encompassing gender identities that are not exclusively masculine or feminine, or that lie outside the traditional gender binary. In the 2015 USTS, up to $35 \%$ of respondents identified as non-binary or genderqueer, an equal number to transgender women and men $(6,7)$. The survey of Minnesota high-schoolers found that about half of TGNB respondents identified primarily as non-binary (6).

Studies evaluating racial and ethnic demographic trends suggest that non-white groups are overrepresented in TGNB populations. Flores et al. estimate transgender prevalence among non-Hispanic whites at approximately 480 per 100,000 , lower than the 770 per 100,000 for nonHispanic blacks, 840 per 100,000 for "Hispanic/Latino" and 640 per 100,000 for "other non-Hispanic" categories (13).

The diverse, evolving language used to describe gender identities can also lend to difficulty with accurate identification of TGNB individuals, particularly for nonbinary individuals. In future efforts to characterize TGNB populations, appropriate identification may be achieved by a two-step approach: by asking individuals their current gender, followed by their sex assigned at birth $(14,15)$. This method avoids pathologizing TGNB status, and in the healthcare setting may facilitate conversations between patients and providers regarding gender identity. Electronic medical record systems have begun to include gender identity questions within their data intake fields, adhering to U.S. Centers for Medicare \& Medicaid Services mandates $(16,17)$.

\section{Epidemiology of transgender health and medical treatment}

Significant health disparities exist for the transgender population and stem from a number of sources: insufficient provider training in healthcare needs specific to TGNB individuals; direct interpersonal or systemic discrimination perpetuated by healthcare providers and institutions; and broader sociopolitical discrimination leading to challenges in finding stable employment and housing, among other chronic stressors $(17,18)$. TGNB identities are associated with increased risk of sexually transmitted infections such as HIV and psychiatric comorbidities such as depression and anxiety when compared to the general population (7).

Gender affirming healthcare is effective and medically necessary for those TGNB individuals who seek it (19). Psychological, hormonal and surgical care for appropriately selected individuals has been unambiguously associated with improvements in gender dysphoria and decreased rates of psychiatric comorbidities (20). A growing number of professional societies have published position statements supporting public and private insurance coverage of gender affirming care (21-25). Provision of such care has historically been compromised by categorical exclusion of transgender-related healthcare by insurers (26). Up to $25 \%$ of transgender individuals who sought hormone therapy coverage were denied by their insurers (7). The Affordable Care Act, however, created a shift away from health insurance that excludes based on preexisting conditions to a coverage specifically for transgender care (27).

Healthcare systems are currently ill-equipped to meet the demand of a growing transgender population. The literature demonstrates numerous instances of transgender individuals being denied medical care related to their gender identity (28). According to 2015 USTS results, $23 \%$ of respondents did not see a doctor when they needed to because of fear of being mistreated as a transgender person (7). Ninety-one percent of TGNB people reported wanting counseling, hormones, and/or puberty blockers, however only $65 \%$ reported ever having any of these (7). Inadequate medical treatment of transgender-related health issues and psychiatric disorders has dire consequences. Serious psychological distress and suicide attempts have been reported at approximately $40 \%$, almost nine times the national suicide rate in the general US population (4.6\%) (29). Seven percent of respondents had attempted suicide in the past year alone (7).

Outside of commonly-discussed transgender health sequelae such as HIV, substance abuse, and psychiatric disorders, relatively little is known about transgenderspecific preventive care needs. For example, ideal regimens for breast, cervical, and prostate cancer screening in TGNB individuals are poorly understood. Longitudinal studies are needed to assess mental and physical health conditions prevalent in the TGNB population.

\section{Growing utilization of gender confirming surgery}

In the most robust survey to date, $25 \%$ of TGNB respondents report having undergone some form of GCS. Other smaller studies report slightly higher ranges (up to 
$35 \%)$, though these are typically carried out in healthcare settings, selecting for those who are accessing care $(7,8)$.

Existing estimates potentially underestimate GCS utilization, due to many of the same factors that limit transgender prevalence studies. GCS procedures are likely under-captured based on ICD-9 or 10 and CPT (Current Procedural Terminology) codes alone, which may not accurately reflect the range of affirming procedures TGBN individuals seek. Many transgender people do not have transgender-related ICD codes or are enrolled as a gender identity other than that with which they identify. In one survey of 122 transgender men, $35 \%$ were enrolled with their insurance providers as female patients (30). Furthermore, many prevalence studies were carried out in previous years, or use aggregate patient populations over several years. These studies report data from periods when GCS was either less common or less frequently recorded. Finally, the wide range of research methods currently employed (online surveys, surgery clinic surveys, claims-based dataset analysis, single institution-reported case volumes) complicate any year-to-year comparison of prevalence.

What is certain, however, is that GCS is a rapidlyexpanding field. Of all procedures recorded by the American Society for Plastic Surgeons (ASPS), GCS was among the most rapidly increasing between 2016 to 2017 (31). GCS increased by $155 \%$ in this period, with a $289 \%$ increase for transgender men and a $41 \%$ increase for transgender women. These rates are significantly increased from the more modest but still marked net 19\% increase in GCS from 2015 to 2016 reported by ASPS.

A number of factors have been identified to influence GCS utilization among transgender people, namely age, income, and race. Older age is associated with increased utilization. One study found a $4 \%$ increased likelihood of having had GCS for each additional year of age (9). Another found a positive association with age over 50 years (32). Affluence was also associated with receipt of GCS. The main effect appears to be proportionally decreased GCS usage with annual income below \$35,000-50,000 (32). There appears to be a relatively smaller effect of increased income over $\$ 50,000$. While only $12 \%$ of transgender people without income and $15 \%$ of people with income less than $\$ 10,000$ per year report having had GCS, $43 \%$ of people with income over $\$ 50,000$ report having had some type of GCS. Black and Latina races appear to be negatively correlated with GCS utilization, although the effect is small and requires further exploration (33).

A number of barriers limit patient access to GCS. Among the most important of these are financial concerns. By one survey, about $25 \%$ of transgender individuals report that the largest barriers to accessing GCS was cost (9). Transgender populations are affected by poverty, unemployment, and lack of insurance at higher rates than the general population (7). Historically, many have been forced to pay out of pocket for GCS, due to categorical exclusions of GCS (26). Still, even after Section 1557 of the Affordable Care Act forbade discrimination on the basis of sex or gender identity, many insured transgender individuals find themselves without coverage for GCS $(27,34)$. As recently as $2015,25 \%$ of transgender individuals reported having difficulty with their insurance providers related to their transgender identity, and over half were denied coverage for GCS (7).

However, insurance coverage of GCS may indeed increase in coming years, due to societal or legal pressures. Financially, expanding coverage of GCS is feasible and beneficial for public health (35). The public health benefits extend beyond just treating gender dysphoria in a small minority of people, but are largely derived from generally decreased rates of several other costly endpoints like HIV, depression, and suicide.

\section{Trends in specific gender confirming procedures}

In general, GCS is more common in transgender men than in transgender women, and least common in gender non-binary or nonconforming populations. Transgender men self-report GCS prevalence at rates of $42-54 \%$, while transgender women report it at around $28 \%$, and non-binary individuals at around 9\% $(7,8)$. By one study, a transgender male identity was associated with an odds ratio for having had GCS of 1.87 , compared with being transgender female (9).

Across transgender populations, chest ("top") surgery is more common than genitourinary reconstructive ("bottom") surgery. Chest surgery is generally reported at about twice the rate of genital GCS. In studies that assessed transgender men and women as an aggregate, chest surgery has been reported at rates between $8-25 \%$, and genital surgery at $4-13 \%(8,9)$. This could be due to a number of factors. Chest surgery may be more important to outward gender expression for many individuals, as the presence or absence of breast tissue is more readily visible in daily life than are the genitalia. Chest surgery is likely more accessible as well, as most plastic surgeons are familiar with breast augmentation and mastectomy for non-gender affirming implications, while relatively few are trained in techniques 
required for transgender genital reconstruction (36).

Genital GCS is generally less common than chest surgery, with prevalence rates of about $25-50 \%$ for transgender men and $5-10 \%$ for transgender women $(7,9,32)$. For transgender women, genital GCS comprises a number of procedures, including vaginoplasty (most commonly intestinal or penile inversion) with labiaplasty and/or clitoroplasty, penectomy, and orchiectomy. Transgender women report bottom surgery at rates between 5-13\% (7-9,32). Even more transgender women desire bottom surgery in the future: between $45-54 \%(7,9)$. Among non-binary people assigned male at birth, $1 \%$ have had vaginoplasty or labiaplasty, and $11 \%$ desire these in the future (7).

Masculinizing genital GCS aims to remove 'female' genitalia (i.e., vagina and labia) and/or create a neophallus. Extirpative procedures such as hysterectomy and salpingooophorectomy appear to be more common than those to reconstruct genital anatomy, which include phalloplasty and metoidioplasty with or without urethral lengthening, scrotoplasty, colpectomy, and penile and testicular implant placement. By USTS 2015 results, hysterectomy is significantly more common than any type of neophalluscreating procedure: $14 \%$ of transgender men have had a hysterectomy, while another $57 \%$ want one in the future (7). Hysterectomy is also relatively common in non-binary people assigned female at birth: $2 \%$ have had it and another $30 \%$ want it in the future.

Phalloplasty and metoidioplasty are the two primary genital reconstructive procedures for transgender male individuals, each with their own urinary, sexual, and aesthetic considerations $(37,38)$. Phalloplasty affords a larger phallus that may allow for standing micturition and penetrative sexual intercourse after urethral lengthening and penile implant placement; however, it is costly, usually requires multiple procedures, has high complication rates, and may result in a stigmatizing donor site scar. Metoidioplasty, or mobilization of the hormonally augmented clitoris with or without urethral lengthening, typically results in a smaller phallus, but can be performed in a single operation with preservation of native tissue sensation. As a whole, less than $5 \%$ of transgender men have had procedures to create a phallus $(8,32)$. One survey distinguished between the surgical options, reporting that $3 \%$ of transgender men have had phalloplasty and $19 \%$ want it in the future, while $2 \%$ have had metoidioplasty and $25 \%$ want it in the future (7).

Transgender women and non-binary individuals assigned male at birth may commonly seek procedures that feminize other highly-gendered aspects of their outward presentation, such as the face, tracheal cartilage, body contour, body hair, and voice. Such procedures constitute $10.6 \%$ of all transgender inpatient hospital visits $(7,8,32,39,40)$. Prevalence rates are about $50 \%$ for hair removal, $3-8 \%$ for facial feminization, and $1 \%$ for feminizing phonosurgery. Prevalence for silicone injections, a risky procedure not endorsed or performed by medical professionals, is around $16.7 \%$, and may have serious or fatal health consequences $(41,42)$. These ancillary procedures are much less common in transgender men (7).

\section{Limitations}

A number of factors specific to TGNB individuals limit estimates of prevalence and healthcare utilization. Transgender demographics are difficult to study, as identifying individuals as transgender can publicly 'out' them, potentially putting them in physical danger or subjecting them to outward discrimination. Therefore, many of the largest studies on transgender epidemiology are anonymous online surveys, which preclude any verification of patients' identities, and preferentially sample those respondents with computers and internet access. Furthermore, many electronic medical records do not record gender identity or sexual orientation data. This makes is difficult to distinguish between GCS and nongender-related indications. For example, such systems could not distinguish between a prophylactic breast cancer mastectomy in a cisgender woman and a gender-affirming top surgery in a transgender man. Lastly, relatively little data, epidemiologic or otherwise, is available on the health needs and practices of non-binary people, which may be quite distinct from those of transgender men and women.

\section{Conclusions}

As visibility of transgender identities have increased in recent years, so too has the prevalence of GCS. Gender affirming care is highly effective for treating gender dysphoria in transgender individuals who seek it, but a number of sociopolitical and economic factors have limited access to care. Further developments in patient-reported outcomes research, transgender-specific provider education, and technical advancements to GCS procedures will all be necessary to meet the healthcare needs of a growing TGNB population $(18,43)$. 


\section{Acknowledgments}

None.

\section{Footnote}

Conflicts of Interest: The authors have no conflicts of interest to declare.

\section{References}

1. Meerwijk EL, Sevelius JM. Transgender Population Size in the United States: a Meta-Regression of Population-Based Probability Samples. Am J Public Health 2017;107:e1-8.

2. Flores AR, Herman JL, Gates GJ, et al. How Many Adults Identify as Transgender in the United States? Los Angeles, CA: The Williams Institute, 2016.

3. Conron KJ, Scott G, Stowell GS, et al. Transgender health in Massachusetts: results from a household probability sample of adults. Am J Public Health 2012;102:118-22.

4. Deutsch MB. Making It Count: Improving Estimates of the Size of Transgender and Gender Nonconforming Populations. LGBT Health 2016;3:181-5.

5. American Psychological Association TFoGIaGV. Report of the Task Force on Gender Identity and Gender Variance. Washington, DC: Author, 2009.

6. Rider GN, McMorris BJ, Gower AL, et al. Health and Care Utilization of Transgender and Gender Nonconforming Youth: A Population-Based Study. Pediatrics 2018. doi: 10.1542/peds.2017-1683.

7. James SE, Herman JL, Rankin S, et al. The Report of the 2015 U.S. Transgender Survey. Washington, DC: National Center for Transgender Equality, 2016.

8. Kailas M, Lu HMS, Rothman EF, et al. Prevalence and types of gender-affirming surgery among a sample of transgender endocrinology patients prior to state expansion of insurance coverage. Endocr Pract 2017;23:780-6.

9. Sineath RC, Woodyatt C, Sanchez T, et al. Determinants of and barriers to hormonal and surgical treatment receipt among transgender people. Transgend Health 2016;1:129-36.

10. Arcelus J, Bouman WP, Van Den Noortgate W, et al. Systematic review and meta-analysis of prevalence studies in transsexualism. Eur Psychiatry 2015;30:807-15.

11. Grant JM, Mottet LA, Tanis J, et al. Injustice at Every Turn: A Report of the National Transgender Discrimination Survey. Washington: National Center for Transgender Equality and National Gay and Lesbian Task
Force, 2011.

12. Herman JL, Flores AR, Brown TNT, et al. Age of Individuals who Identify as Transgender in the United States. Los Angeles, CA: The Williams Institute, 2017.

13. Flores AR, Brown TNT, Herman JL. Race and Ethnicity of Adults who Identify as Transgender in the United States. Los Angeles, CA: The Williams Institute, 2016.

14. Deutsch MB, Buchholz D. Electronic health records and transgender patients--practical recommendations for the collection of gender identity data. J Gen Intern Med 2015;30:843-7.

15. Tate CC, Ledbetter JN, Youssef CP. A two-question method for assessing gender categories in the social and medical sciences. J Sex Res 2013;50:767-76.

16. Alper JF, Feit MN, Sanders JQ. Collecting Sexual Orientation and Gender Identity Data in Electronic Health Records: Workshop Summary. Washington (DC): National Academies Press (US), 2013.

17. Medicare and Medicaid Programs; Electronic Health Record Incentive Program-Stage 3 and Modifications to Meaningful Use in 2015 Through 2017. 2015.

18. Dubin SN, Nolan IT, Streed CG Jr, et al. Transgender health care: improving medical students' and residents' training and awareness. Adv Med Educ Pract 2018;9:377-91.

19. Coleman E, Bockting W, Botzer M, et al. Standards of Care for the Health of Transsexual, Transgender, and Gender-Nonconforming People, Version 7. Int J Transgend 2012;13:165-232.

20. White Hughto JM, Reisner SL. A Systematic Review of the Effects of Hormone Therapy on Psychological Functioning and Quality of Life in Transgender Individuals. Transgend Health 2016;1:21-31.

21. Hembree WC, Cohen-Kettenis PT, Gooren L, et al. Endocrine Treatment of Gender-Dysphoric/ Gender-Incongruent Persons: An Endocrine Society Clinical Practice Guideline. J Clin Endocrinol Metab 2017;102:3869-903.

22. The American Medical Association. Removing Financial Barriers to Care for Transgender Patients. H-185.950; 2016.

23. WPATH (World Professional Association for Transgender Health). Recommended Benefits Policy Document: Created in Partnership with Starbucks. 2018. Available online: https://stories.starbucks.com/stories/2018/theyare-lifesaving-starbucks-offers-expanded-benefits-fortrans-people/

24. ACOG (American College of Obstreticians and 
Gynecologists). Health Care of Transgender Individuals. In: Committee on Healthcare for Underserved Women. 2011. Available online: https://www.acog.org/-/media/ Committee-Opinions/Committee-on-Health-Care-forUnderserved-Women/co512.pdf

25. WPATH. Position Statement on Medical Necessity of Treatment, Sex Reassignment, and Insurance Coverage in the U.S.A. WPATH Policy Statements 2016. Available online: https://www.wpath.org/newsroom/medicalnecessity-statement

26. Gage SE. The transgender eligibility gap: how the ACA fails to cover medically necessary treatment for transgender individuals and how HHS can fix it. New England Law Review 2015;49:42.

27. Nondiscrimination in Health Programs and Activities: Section 1557 of Affordable Care Act. Available online: https://www.federalregister.gov/ documents/2016/05/18/2016-11458/nondiscriminationin-health-programs-and-activities?utm_campaign=su bscription+mailing+list\&utm_medium=email\&utm_ source=federalregister.gov

28. Ben-Asher B. The Necessity of Sex Change: A Struggle for Intersex and Transsex Liberties. Harvard Journal of Law and Gender 2006;29(51).

29. NIMH (National Institute of Mental Health). Mental Health Statistics: Suicide. 2018. Available online: https:// www.nimh.nih.gov/health/statistics/suicide.shtml

30. Rachlin K, Green J, Lombardi E. Utilization of health care among female-to-male transgender individuals in the United States. J Homosex 2008;54:243-58.

31. ASPS (American Society of Plastic Surgeons). Plastic Surgery Statistics Report. 2017. Available online: https:// www.plasticsurgery.org/documents/News/Statistics/2017/ plastic-surgery-statistics-full-report-2017.pdf

32. Beckwith N, Reisner SL, Zaslow S, et al. Factors associated with gender-affirming surgery and age of hormone therapy initiation among transgender adults. Transgend Health 2017;2:156-64.

33. Wilson EC, Chen YH, Arayasirikul S, et al. Connecting the dots: examining transgender women's utilization

Cite this article as: Nolan IT, Kuhner CJ, Dy GW. Demographic and temporal trends in transgender identities and gender confirming surgery. Transl Androl Urol 2019;8(3):184190. doi: 10.21037/tau.2019.04.09 of transition-related medical care and associations with mental health, substance use, and HIV. J Urban Health 2015;92:182-92.

34. US Department of Health and Human Services. Access to healthcare: nondiscrimination. 2017. Available online: https://www.hhs.gov/programs/topic-sites/lgbt/ accesstohealthcare/nondiscrimination/index.html

35. Padula WV, Heru S, Campbell JD. Societal Implications of Health Insurance Coverage for Medically Necessary Services in the U.S. Transgender Population: A CostEffectiveness Analysis. J Gen Intern Med 2016;31:394-401.

36. Dy GW, Osbun NC, Morrison SD, et al. Exposure to and Attitudes Regarding Transgender Education Among Urology Residents. J Sex Med 2016;13:1466-72.

37. Dy GW, Sun J, Granieri MA, et al. Reconstructive Management Pearls for the Transgender Patient. Curr Urol Rep 2018;19:36.

38. Frey JD, Poudrier G, Chiodo MV, et al. A Systematic Review of Metoidioplasty and Radial Forearm Flap Phalloplasty in Female-to-male Transgender Genital Reconstruction: Is the "Ideal" Neophallus an Achievable Goal? Plast Reconstr Surg Glob Open 2016;4:e1131.

39. Canner JK, Harfouch O, Kodadek LM, et al. Temporal Trends in Gender-Affirming Surgery Among Transgender Patients in the United States. JAMA Surg 2018;153:609-16.

40. Morrison SD, Vyas KS, Motakef S, et al. Facial Feminization: Systematic Review of the Literature. Plast Reconstr Surg 2016;137:1759-70.

41. Wilson E, Rapues J, Jin H, et al. The use and correlates of illicit silicone or "fillers" in a population-based sample of transwomen, San Francisco, 2013. J Sex Med 2014;11:1717-24.

42. Leonardi NR, Compoginis JM, Luce EA. Illicit Cosmetic Silicone Injection: A Recent Reiteration of History. Ann Plast Surg 2016;77:485-90.

43. Andréasson M, Georgas K, Elander A, et al. PatientReported Outcome Measures Used in Gender Confirmation Surgery: A Systematic Review. Plast Reconstr Surg 2018;141:1026-39. 